\title{
ARENA 2.0: The next generation automated remote environmental navigation apparatus to facilitate cross-species comparisons in behavior and cognition
}

\author{
Julia Schroeder $^{1}$ • Dennis Garlick ${ }^{1}$ - Aaron P. Blaisdell ${ }^{1}$
}

Published online: 2 June 2017

(C) Psychonomic Society, Inc. 2017

\begin{abstract}
A series of experiments illustrated the effectiveness and flexibility of a newly developed Automated Remote Environmental Navigation Apparatus (ARENA) as an alternative to traditional operant and open-field procedures. This system improves the concept developed by Badelt and Blaisdell (Behavior Research Methods, 40, 613-621, 2008; see also Leising, Garlick, Parenteau, \& Blaisdell in Behavioural Processes, 81, 105-113, 2009), with significant upgrades in flexibility and reliability, as well as a reduction in cost. ARENA is particularly well adapted for open-field studies and eliminates many confounding factors associated with traditional procedures, such as handling effects and physical cues left by the subject. The original system was based on wireless modules with a small stimulus-response well. Nosepokes or pecks within the aperture of the well could be detected and recorded by a computer. ARENA 2.0 increases the flexibility of this system by replacing the modules with stimulus presentation through a data projector mounted on the ceiling and response detection and recording through a video camera system. We report the specifics of this system as well as behavioral tests using rats and pigeons. We demonstrated the feasibility of ARENA 2.0 for the acquisition of conditional approach to a visual target, followed by tests showing generalization of performance to novel locations and visual properties of the target. These experiments support the use of this technology for automated tasks traditionally studied through open-field preparations or using touchscreen-equipped operant chambers. The advantages of ARENA 2.0 over the
\end{abstract}

Aaron P. Blaisdell

blaisdell@psych.ucla.edu

1 Department of Psychology, University of California, Los Angeles, 1285 Franz Hall, Los Angeles, CA 90095-1563, USA original system are a significant reduction in cost and increased reliability, ease of use, and flexibility in both stimulus configuration and subject response measures.

Keywords Operant · Open field · Comparative · Automation $\cdot$ Behavior $\cdot$ Cognition

Science is improved through the use of objective and repeatable forms of measurement. Animal psychology research has greatly benefited from developments in the standardization and automation of recording behavior. Most notable of these was the invention of the Skinner box, which allowed lever-pressing or key-pecking to be recorded without the need of direct human observation or interference (Skinner, 1938, 1956). This invention greatly reduced the time it took to administer experiments, while also allowing more direct comparison of studies across laboratories. Despite its advantages, the Skinner box is an artificial environment that falls short of a perfect simulation of the test subject's natural environment. As such, the behaviors elicited in a Skinner box may be constrained relative to behaviors in the natural environment. This limits the ecological validity and generalizability of results collected in the Skinner box.

Behavior in a Skinner box typically involves unrestricted emission of an operant, such as pressing a lever or pecking a key. Other forms of apparatus that utilize operant techniques, such as the touchscreen-equipped operant chamber, have expanded on the utility of the traditional Skinner box by providing for richer stimulus displays and less constrained targets for response. These tools have been particularly useful for crossspecies comparisons, primarily among pigeons, rats, and primates (e.g., Blough, 1986; Cook, Geller, Zhang, \& Gowda, 2004; Elsmore, Parkinson, \& Mellgren, 1989). Touchscreenequipped operant chambers offer the ability to present a wide range of stimuli in varying locations on the screen, allowing 
for the investigation of spatial cognition as well as visual perception (e.g., Cook, Katz, \& Blaisdell, 2012; Cook, Shaw, \& Blaisdell, 2001; Sawa, Leising, \& Blaisdell, 2005; Scarf, Hayne, \& Colombo, 2011; Stahlman, Roberts, \& Blaisdell, 2010; Spetch, Cheng, \& Mondloch, 1992; Young, Wasserman, \& Garner, 1997). They avoid the need to physically move visual stimuli and spatial cues between trials, because virtual stimuli can be effortlessly presented at any location on the screen, in static or dynamic format, both between trials or even within a trial. This eliminates the influence of scent cues or other disruptive changes that may result from physical interventions. Touchscreen procedures are also similar to the Skinner box in that they allow responses to be recorded using a mechanism that is not dependent on human observation.

Nevertheless, the touchscreen methodology presents certain limitations for studying learning and cognition. Subject response, although less constrained in space than the traditional Skinner box equipped with a response lever or key, is still relatively restricted to the small dimensions of the touchscreen surface. Likewise, responses consist only of interactions with the surface of the touchscreen (pecking or touching) and not movement of the animal through space. This limits the utility of an operant touchscreen procedure as compared to methodologies that allow the subject to move through space, such as a maze or open field (though results from spatial tasks presented on the touchscreen have in some cases been shown to mirror those collected using open field procedures; e.g., Leising \& Blaisdell, 2009; Spetch, Cheng, \& MacDonald, 1996). Thus, there is a need for the development of a more suitable automated apparatus for the collection of operant and Pavlovian behavior but that allows the subject free movement through space. This new apparatus should allow the animal to physically move through space, and record information about subject location across time, as well as the spatial location of choice behavior.

An automated open-field operant task would also enable the study of small-scale navigation and spatial cognition. Spatial navigation is a defining feature of the animal kingdom, with a diverse array of species using surprisingly similar methods of navigation (Capaldi, Robinson, \& Fahrback, 1999; Leising \& Blaisdell, 2009). Even nonanimals such as slime molds have mechanisms to support efficient navigation (Nakagaki, Yamada, \& Tóth, 2000). Laboratory studies of spatial cognition utilize more naturalistic procedures and settings for spatial behavior, such as navigating in an open field, looking for food buried in a sandbox, or swimming to find a hidden platform so as to escape a water bath (Shettleworth, 1998). These procedures have certain drawbacks, however, when compared to operant procedures. These limitations include a lower volume of data throughput, direct human involvement that may be disruptive to the animal, introduction of unintended confounding influences (e.g., scent cues, scuff marks, etc.), and the presence of rewards in the enclosure that can cue the subject independently from the intended discriminative stimuli. Moreover, reliance on human scoring of behavior opens the opportunity for biases and lapses in attention to skew data collection (e.g., Hauser, Weiss, \& Marcus, 2002). Nearly all open field procedures require the animal to be captured after every trial in order to reset the field, disrupting the animal's natural foraging or navigation behaviors, the very behaviors most users of appetitive spatial tasks are seeking to study. The reliance on a human experimenter to physically set the apparatus and handle the animal before each trial limits the volume of data collection and the validity of the results collected. Finally, the touchscreen is not an ideal technology for use with true navigation or the study of egocentric spatial behavior, which is fixed with respect to stimuli in the touchscreen operant box, but can vary in an open field setup.

To overcome these limitations, Badelt and Blaisdell (2008) developed the use of capacitive proximity sensor technology for measuring subject behavior in space. This technology was later incorporated into an ARENA (Automated Remote Environmental Navigation Apparatus) system based on wireless modules (Leising, Garlick, Parenteau, \& Blaisdell, 2009). The modules were square plastic devices that could serve as targets or landmarks. Each module had a circular well in its top center fitted with a capacitive proximity sensor. When part of the animal (a beak, paw, nose, or finger) comes close enough to the sensor surface located in the cup, the computer records the resulting change in capacitance of the sensor as a response. Colored LED lights located within each module allowed them to also serve as visual cues, with the ability to present different colors of light through computer control. This system approximated the free-operant techniques initially developed by Skinner, and allowed many trials to be conducted without disrupting or interrupting subject behavior. Additionally, different trial types could be presented between trials as the LED lights allowed the modules to indicate different types of landmarks or other discriminative stimuli. Leising et al. (2009) demonstrated the usefulness of this technology for the study of simple instrumental control by a visual cue, as well as a visual discrimination, and conditional discrimination in pigeons. We have also used ARENA to study associative-learning processes in spatial behavior, such as blocking (Leising, Wong, Ruprecht, \& Blaisdell, 2014), overshadowing (Leising, Garlick, \& Blaisdell, 2011), and simple food choice using spatially discrete choice responses (Biedermann, Garlick, \& Blaisdell, 2012).

Nevertheless, whereas this system did overcome many of the previously noted shortfalls of nonautomated open-field apparatuses and the automated touchscreen system, it was found to suffer from problems of its own. Each module had to be physically placed within the room, and only those locations could be used as the targets of responses and for stimulus presentation. Moreover, the equipment was lacking in reliability. The modules themselves were prone to damage from rats chewing on them, animal debris often interfered with the 
sensors (multiple times per week), and the wireless transmitters were heavy energy users, necessitating the frequent changing of batteries (typically every $2-3$ days). Despite implementing a strict in-house protocol of scheduled battery changes, modules became unresponsive during a session of data collection on average three to four times per week, which compromised the value of the data collected in those sessions. Beyond these issues, a further drawback was the need to design and construct complex circuit boards, necessitating expertise in engineering and deep-level programming. Once designed, printing the circuit boards was outsourced and not cheap for small-scale production. At approximately $\$ 300$ per printed board, the system of 16 modules used in our lab cost close to $\$ 5,000$, not counting batteries, which needed continual replacement.

Here we present an updated ARENA system (ARENA 2.0) that permits even greater flexibility in the arrangement of spatial cues and target locations than the previous version, while limiting the need for complicated, specially designed custom hardware. The system consists of a data projector mounted on the ceiling of the open-field room, a video camera mounted near the projector to record subject behavior, a reward delivery device (e.g., a food hopper), and a personal computer with which to control stimulus display, audio delivery, and reward delivery, and to record subject behavior in real time. The essence of the approach is the projection of a visual display on the floor of the ARENA from above, and the recording of a subject's response as it interacts with the display. The system we test in this report was controlled by custom software written in Microsoft Visual C\# (freely available on our laboratory website: LINK). It might be possible to program this system using simpler programs or GUIs, such as MATLAB, E-Prime, Noldus, Anymaze, and so forth. But the custom program developed in our lab in MS Visual C\# is designed to allow the data projector and feedback from the video camera to interact. This allows for controlling stimulus events in real time in response to subject behavior (e.g., by blinking an image on and off to mark a correct response when the subject chooses that stimulus). Any video-tracking software that (a) can respond in real time to subject behavior and (b) is not negatively affected by small or wholesale changes in the background projected on the arena floor should in theory work with ARENA 2.0.

The software developed in our lab takes an image from the video feed before the experiment commences, while the animal is not present. ${ }^{1}$ The computer then continuously compares the video input at specified locations relative to this baseline image. If the number of pixels that deviate from baseline in the red, green, and blue dimensions exceeds a threshold set as the baseline, then the program records the animal as being present at the

\footnotetext{
${ }^{1}$ The experimental program and sample files containing the raw data, in Excel format, and accompanying videos are available online at the following link: http://blaisdell.wixsite.com/aaronblaisdell/supplementary-materials.
}

location. This allowed the recording of choice behavior in real time as the subject interacts with a static or dynamic stimulus display.

Furthermore, we can vary the length of time the subject is required to remain within the target location before a choice is recorded. This minimizes false alarms created by the subject passing through a target region while not explicitly making a choice. Thus, choice behavior can be made deliberative. Timing accuracy is superior to that of a human-operated hand timer and can be changed at a very fine scale between trials, allowing for alteration of the dependent measure within a session. Unlike traditional open-field methods, spatial cues and target cues are projected from the ceiling, allowing the presentation and removal of the cue contingent on subject response. We can also provide visual (e.g., flashing the target cue off and on when chosen) and auditory (e.g., a "beep" from a nearby speaker) feedback to mark stimulus choice or provide secondary reinforcement.

This system has many advantages over the original ARENA system, in that it does not require any physical modules to be present. It can automate control over the visual display in real time, including dynamic and interactive displays. The types of visual information that can be presented are much more diverse. And the system requires only a standard computer, data projector, video camera, and reward delivery system, all of which are relatively cheap and easy to obtain from commercial providers. An adequate data projector costs about $\$ 100-\$ 200$, and a camera is about $\$ 20-\$ 40$. Thus the whole system, not including computer, monitor, keyboard, mouse, and wires, costs no more than about $\$ 240$, as compared to the $\$ 5,000$ described above for an array of 16 ARENA 1.0 modules. ARENA 2.0 no longer requires sensors at target locations for response detection, thereby allowing for a greater number of potential target sites to be used. In the new system, a camera mounted on the ceiling in the center of the room records subject behavior in real time, and rapid image processing allows for detection of the subject responses to target locations. The use of completely digital image display technology avoids any disruption created by subject handling, physical setup between trials, and inadvertent odor cues. Rewards are provided at a single location (a food hopper) in a small enclosure adjacent to the ARENA. A completely automated system allows for true operant, as well as Pavlovian, behavior. To examine the effectiveness of this system, we tested pigeons and rats on a number of tasks that were designed to demonstrate the flexibility and utility of the system.

\section{Experiment 1}

This experiment examined the ability of a single visual target, in this case an illuminated white circle always presented at the center of the ARENA floor, to elicit an instrumental response 
in both rats and pigeons. Each subject was first trained to approach the target. If the subject remained at the target location ("dwell time") for $0.2 \mathrm{~s}$, a food reward was delivered. Once the subject was reliably earning rewards with a dwell time of $0.2 \mathrm{~s}$, the length of time the subject was required to remain within the target location was increased by $0.05 \mathrm{~s}$ with every successful trial, and deceased $0.005 \mathrm{~s}$ with every unsuccessful attempt. This staircasing procedure was used to determine the maximum dwell time each subject could maintain. After a subject's maximum dwell time was determined, we varied the location in the ARENA at which the target stimulus was presented, to determine whether responding was controlled by the visual stimulus or the room location.

\section{Method}

\section{Subjects}

Three female pigeons (Columba livia) and eight female LongEvans rats (Rattus norvegicus) served as subjects. The pigeons, which had prior experience in touchscreen-operant tasks, were individually housed in stainless steel home cages with wire mesh floors in a vivarium maintained on a 12/12-h light/dark cycle. Experiments were conducted during the light portion of the schedule. The rats, which had prior experience in Pavlovian conditioning chambers, were pair-housed in transparent plastic tubs with a wood-shaving substrate in a vivarium maintained on a 14/10-h dark/light cycle. Experiments were conducted during the dark portion of the schedule. All subjects were under a food restriction schedule and were maintained at $85 \%$ of their free-feeding weight, but they were allowed free access to water (and grit, for pigeons) while in their home cages.

\section{Apparatus}

Experimental manipulations took place in an anechoic room with a test area of $2.3 \times 1.5 \mathrm{~m}$ (Fig. 1). The walls of the room were covered in white sound-insulating tiles. The east wall of the room had a full-sized door. On the south wall was an access door for wires connecting to a PC in an adjacent room. The door that separated the test area from the start box was $31.8 \mathrm{~cm}$ high and $16.7 \mathrm{~cm}$ wide. The bottom of the door was $3.8 \mathrm{~cm}$ above the floor and was located $78.0 \mathrm{~cm}$ from the west wall and $112.0 \mathrm{~cm}$ from the east wall. When testing pigeons, uniform illumination was provided by four $25-\mathrm{W}$ incandescent lights mounted on shower rods positioned $10.2 \mathrm{~cm}$ below the ceiling. The lights faced upward, to provide indirect lighting. The background illumination remained off when testing the rats. A white-noise generator elevated $109.2 \mathrm{~cm}$ from the floor and centrally located above the automated door delivered an oscillating 56-63 $\mathrm{dB}(\mathrm{A})$ background noise. Two HarmanKardon multimedia speakers were mounted at the same height. A logitech VU-0011 color video camera with wideangle lens was mounted on the ceiling $2.4 \mathrm{~m}$ above the floor of the test area and allowed for viewing and recording experimental procedures in the adjacent room. A BenQ ms612ST data projector was mounted from the wall containing the start box door.

A hopper was mounted on the rear (south) wall of a holding cage, with the hopper opening located $10 \mathrm{~cm}$ above the floor of the cage. The holding cage was located on the other side of the automatic door from the test area. It measured 35.6 $\times 36.8$ $\times 30.5 \mathrm{~cm}$, and was made of plywood boards for rats and white Perspex for pigeons. When a reward was initiated, the speakers emitted a 0.5 -s digital noise (Windows XP xclamation.wav, $69 \mathrm{db}(\mathrm{A})$ ), and the hopper delivered a single 45-mg sucrose pellet (TestDiet F0042), for rats, or $3 \mathrm{~s}$ of access to grain, for pigeons, starting from when the pigeon placed its head in the hopper, thereby interrupting an infrared photo beam projecting across the hopper opening. A target stimulus consisted of a white circle, $9.5 \mathrm{~cm}$ in diameter unless otherwise noted, projected onto the ARENA floor from the data projector. Possible target locations were arranged in a $5 \times$ 5 array, with their centers located $20 \mathrm{~cm}$ apart, starting $51 \mathrm{~cm}$ from the wall opposite the door and $21.5 \mathrm{~cm}$ from the wall opposite the start box (see Fig. 1).

\section{Procedure}

Cage exposure Each rat was fed ten sucrose pellets from tweezers by the experimenter for two days while in its home cage, to familiarize the rat with the reward. Pigeons were familiar with the grain reward, since it was their regular diet.

Hopper training The first phase of training familiarized the subjects with the food hopper. The door between the ARENA and the holding cage was blocked during hopper training so that the subject was confined to the holding cage. At the beginning of each session, the subject was placed in the holding cage facing the food hopper. A reward was delivered on a 30-s fixed-time (FT-30) schedule during each 10-min session. To be considered to have passed hopper training, a subject was required to have consumed all pellets from the hopper by the end of the session for two consecutive sessions. Up to four sessions were performed daily, with an intersession interval of $10 \mathrm{~min}$.

Phase 1: Initial target training The session started with the subject being placed in the holding cage. The door to the ARENA remained open. A single location in the middle of the array was the only location used in Phases 1 and 2 of target training. The stimulus was projected at a location $57 \mathrm{~cm}$ from the center of the door to the holding cage. The subject was allowed to 


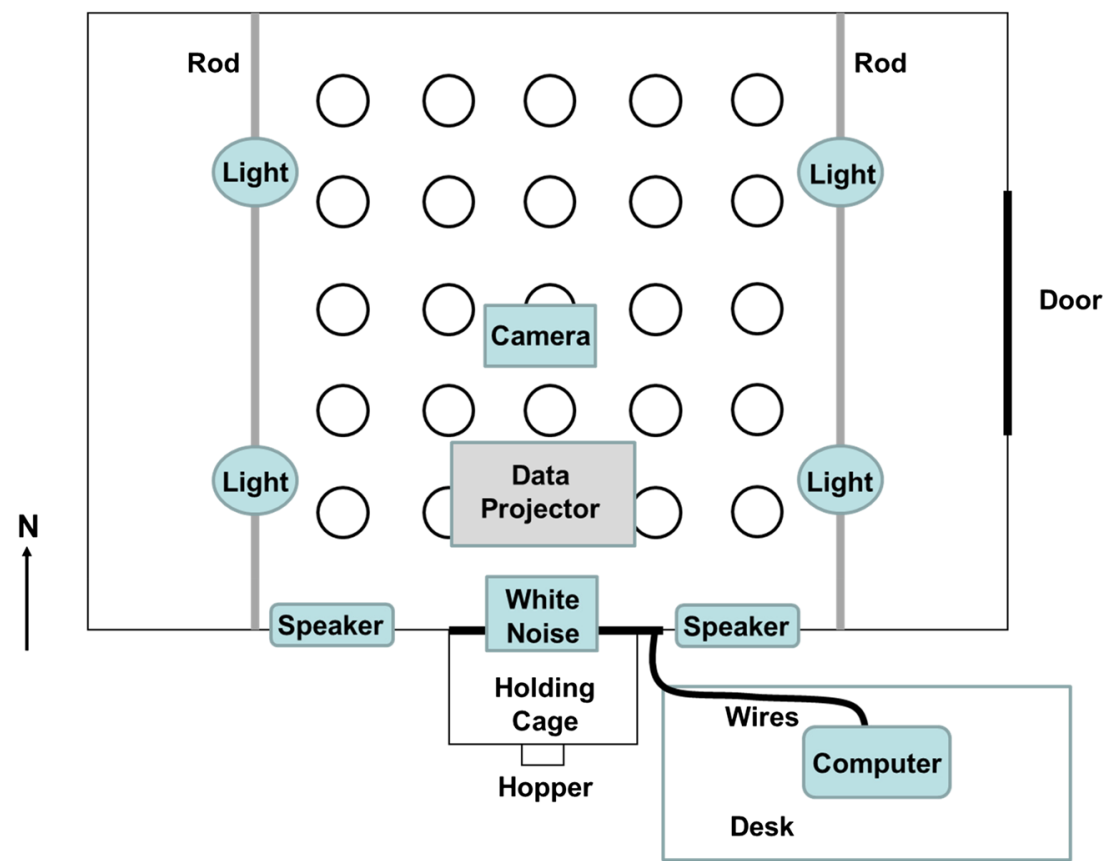

Fig. 1 ARENA 2.0 schematic, top-down view, with a $5 \times 5$ grid of possible stimulus locations. Items in gray are raised above the floor. Items in white are on the floor

explore the ARENA. Any time the subject remained in the illuminated location for $0.2 \mathrm{~s}$, the target was turned off, the tone was played, and a reward was delivered to the hopper in the holding cage. If no response was made within $120 \mathrm{~s}$, the target was turned off, but no reward was delivered. In either case, the next trial would then start after a 30 -s intertrial interval (ITI). Sessions in this and all other phases lasted $30 \mathrm{~min}$, and each animal received one session per day. To advance to Phase 2 , the subject was required to visit the target a minimum of 15 times during a session and to have consumed all of the rewards delivered to the hopper.

Phase 2: Dwell time manipulation Phase 2 was identical to Phase 1, except that the time for which the subject was required to remain within the target (dwell time) was adjusted according to a staircase procedure. This staircase worked on a 0.05 -s increase following a successful visit to the target, with a 0.005 -s decrease when no successful visit occurred and the trial timed out. Once a subject's dwell time had plateaued, the staircase procedure was terminated. Pigeon subjects showed no evidence of reaching a dwell time limit; thus, rather than continue the experiment indefinitely, a subject was considered to have completed this phase once it exceeded a dwell time of $10 \mathrm{~s}$.

Phase 3: Multiple target location training In Phase 3, the procedure was adjusted to test for generalization of discriminative control of the instrumental response by the target. The procedure consisted of placing the target at novel locations on the ARENA floor. Phase 3 was identical to Phase 2, with the exception that the target was presented at various locations selected randomly from the set of 25 possible target locations in the array (Fig. 1). The subject was required to remain at the target location for $1 \mathrm{~s}$ for reinforcement. Sessions lasted 30 min, during which subjects could complete up to 40 trials.

\section{Results and discussion}

Phase 1: Initial target training Two of the pigeons reached the criterion performance of stimulus approach in three sessions, and the third bird took four sessions to reach criterion. Five of the rats took two sessions, and three rats took three sessions to reach criterion approach performance. Thus, there was no difference in the rapid acquisition of approach behavior, and there was no need to hand-shape approach behavior.

Phase 2: Dwell time manipulation Pigeons $(M=11.18 \mathrm{~s}$, $S D=1.01)$ were able to stay on the target location significantly longer than were rats $(M=5.68 \mathrm{~s}, S D=$ 1.99) by the end of Phase 2, $t(9)=-4.50, p<.001, g=$ 3.046 (Fig. 2). 


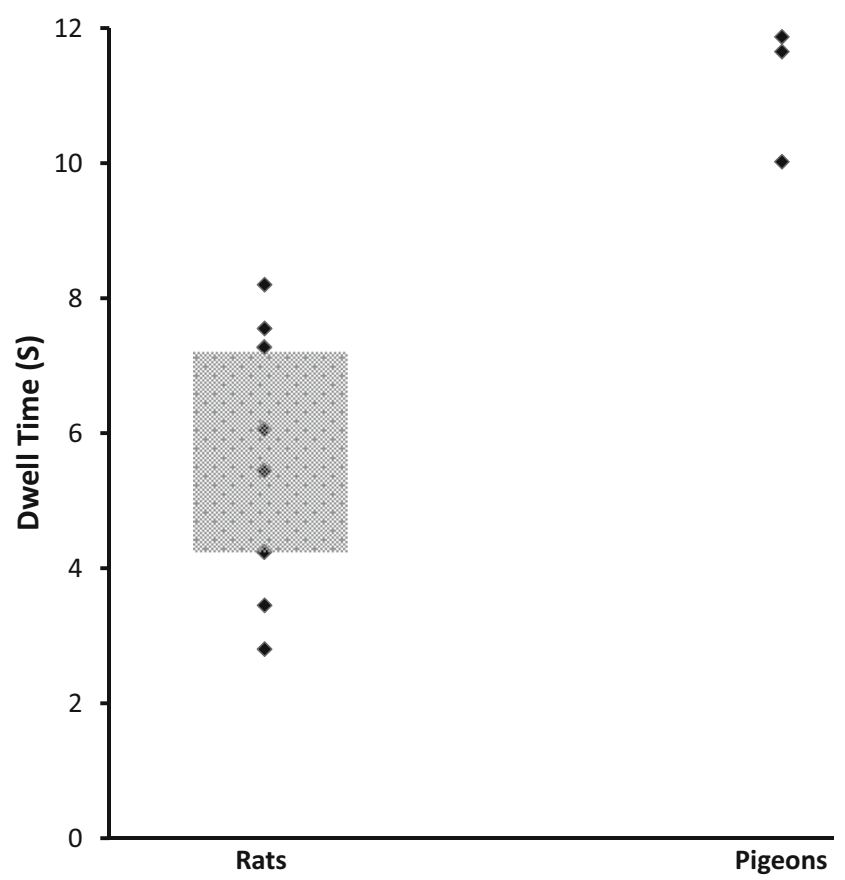

Fig. 2 Dwell times from Experiment 1, Phase 2, for each of the eight rats and three pigeons. The dark box indicates the $95 \%$ confidence interval for dwell times in the rats. Since the pigeons were halted before reaching their asymptotic dwell time, no such calculation was performed on their data

Phase 3: Multiple target location training All subjects generalized responding to novel target locations (Fig. 3, top panel). A mixed analysis of variance (ANOVA) conducted on response times with species as a between-subjects variable and distance from the training location as the repeated measure revealed a main effect of species, $F(1,9)=95.45, p<$ $.001, \eta^{2}=.631$, but no effect of distance, $F(1,9)=2.71, p>$ .05 , nor an interaction $F<1$. Pigeons were much faster at completing a trial than were rats. The bottom panel of Fig. 3 shows that the response times to each location during Phase 3 did not differ from the mean response times to the target location in Phase 2. Thus, the cue-mediated performance of both rats and pigeons generalized perfectly to the novel stimulus locations.

Both species learned to remain within the target area for an extended period of time. This was important because it allowed the automated detection procedure to reliably distinguish subject choice from the mere passage through a location. Nevertheless, there was a dramatic species difference in the time that each species was able to remain on the target location. These differences are likely due to differences in foraging strategies. Pigeons tend to stand in a single location and consume all the food in their reach. Rats, on the other hand, tend to bring food to a safe location for consumption. Because our room was a large open space, the extended dwell times may have been a less natural behavior for the rat foraging system. By increasing the time criterion necessary for reward, the cost of a selection can be manipulated within an experiment to titrate to a value that minimizes false alarms while maintaining a high degree of hits.

Likewise, for both species, control of the behavior acquired during Phase 2 was by the target's visual properties and not by the target location, which was fixed during Phase 2. Placing the target at novel locations in Phase 3 resulted in subjects approaching the target in those novel locations and not spending an inordinate amount of time at the location used in Phase 2. This is not surprising for pigeons, given that they are visually guided foragers, but was a bit more surprising for rats, given the dominant role of spatial features in much of the rat foraging literature.

\section{Experiment 2}

Testing at multiple locations ensured that the visual target used in Experiment 1 was salient to subjects of both species, since they readily generalized responding to novel locations, and that the visual target could elicit choice behavior at novel locations without the need for locationspecific training.

In Experiment 2, we further investigated how subjects would generalize to four other visual dimensions of the target. This was important because many psychological tests require using novel stimuli, features, or configurations of stimuli. Thus, the aim of Experiment 2 was to determine how flexible was the instrumental approach to the target established through the training procedures of Experiment 1. To examine generalization across different stimulus dimensions, the stimulus was separately varied in terms of size, brightness, color, and texture (a stimulus set is shown in Fig. 4). Each novel transformation of one of these dimensions was tested on a separate trial consisting of an individual presentation of the transformed stimulus. Upon completion of individual testing of all novel stimuli, subjects were then tested for their preferences among the stimuli by presenting them with nondifferentially rewarded trials of five randomly selected stimuli that had been previously tested individually.

\section{Method}

\section{Subjects and apparatus}

The same subjects and apparatus from Experiment 1 were used in Experiment 2. The only exception was that the target stimulus was systematically manipulated along the dimensions of size, brightness, pattern, and color (see examples in Fig. 4). Only one dimension was varied at a time, with no combinations of more than one variation 

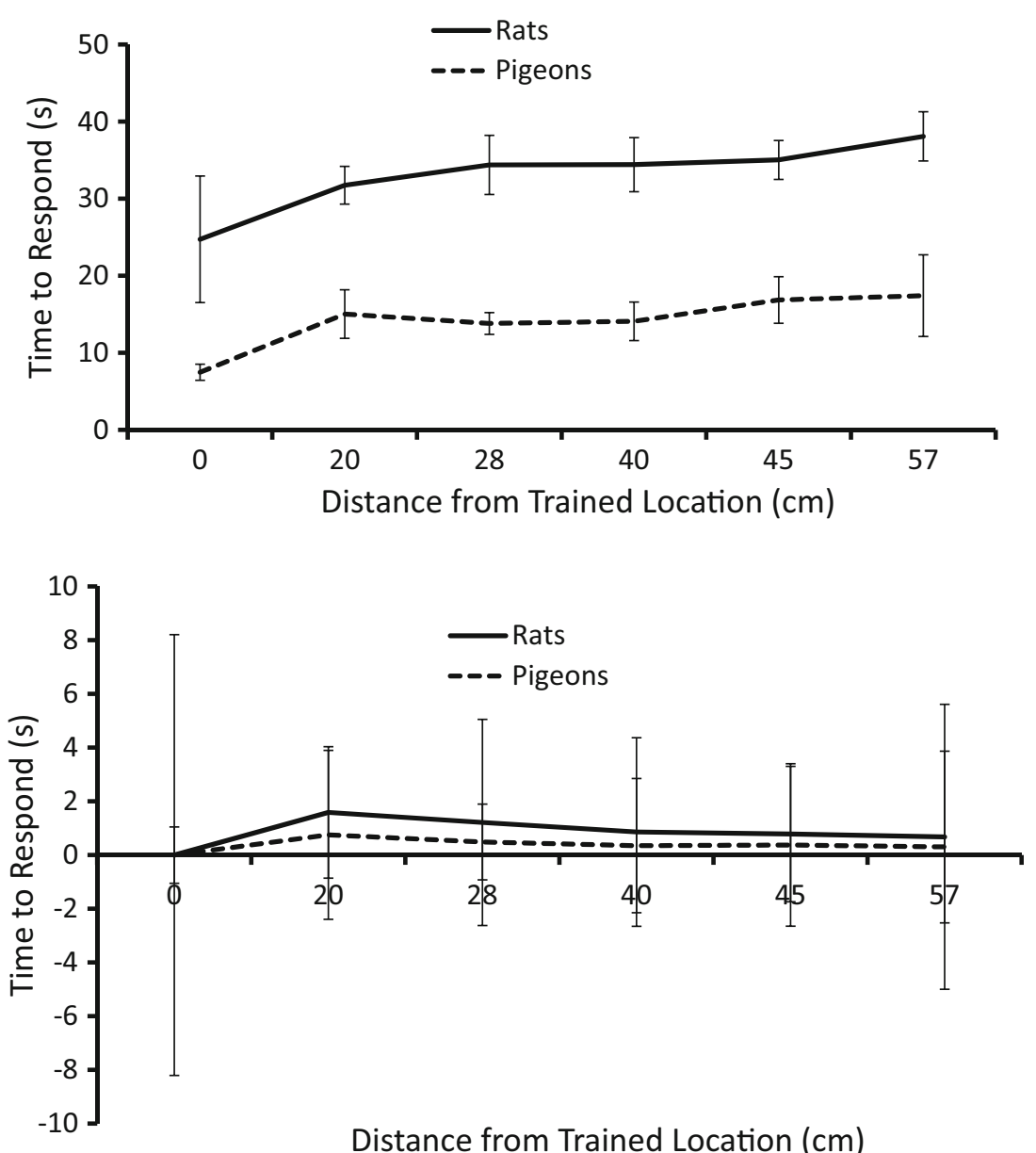

Fig. 3 Top panel: Mean absolute response times, in seconds, to select target locations in Experiment 1, Phase 3. Bottom panel: Mean relative response times to each stimulus location during Phase 3, relative to the

simultaneously. Experiment 2 began the day after subjects had completed Experiment 1. Size was reduced from the training value of $9.5 \mathrm{~cm}$ to values of $7.5,5$, and $2.5 \mathrm{~cm}$ diameter. Brightness was changed from a training value of $100 \%$ to values of $75 \%, 50 \%$, and $25 \%$ of the training target brightness. The color was changed from the white used in training to blue, red, green, and yellow. Texture was changed from solid, as in training, to LargeGrid, DarkUpwardDiagonal, Plaid, and SolidDiamond, as defined in the Microsoft Visual C\# library.

\section{Procedure}

Stimulus generalization test Selection from among the 14 novel stimuli and the original stimulus was randomized without replacement within 15 trial blocks. Up to three blocks were presented within each 30-min session. The location of presentation was randomly selected from within the locations used in Experiment 1, Phase 3. mean response times during Phase 2. Training location $=0$. Error bars denote $95 \%$ confidence intervals

Any time the subject remained in the illuminated location for $1 \mathrm{~s}$, the target was turned off, the tone was played, and a reward was delivered to the hopper in the holding cage. If no response was made within 120 $\mathrm{s}$, the target was turned off, but no reward was delivered. Five generalization sessions were conducted per subject.

Feature preference testing To determine the relative weights with which stimulus features exerted control over responding, a stimulus preference test was conducted. As in Experiment 1, subjects were required to remain within the illuminated location for $1 \mathrm{~s}$ before a reward was presented and the tone sounded. The complete set of stimuli was randomized into sets of five without replacement, so that each stimulus was tested with each other stimulus an equal number of times. For each trial, a set of five stimuli was presented in a semicircular arrangement, such that all stimuli were 


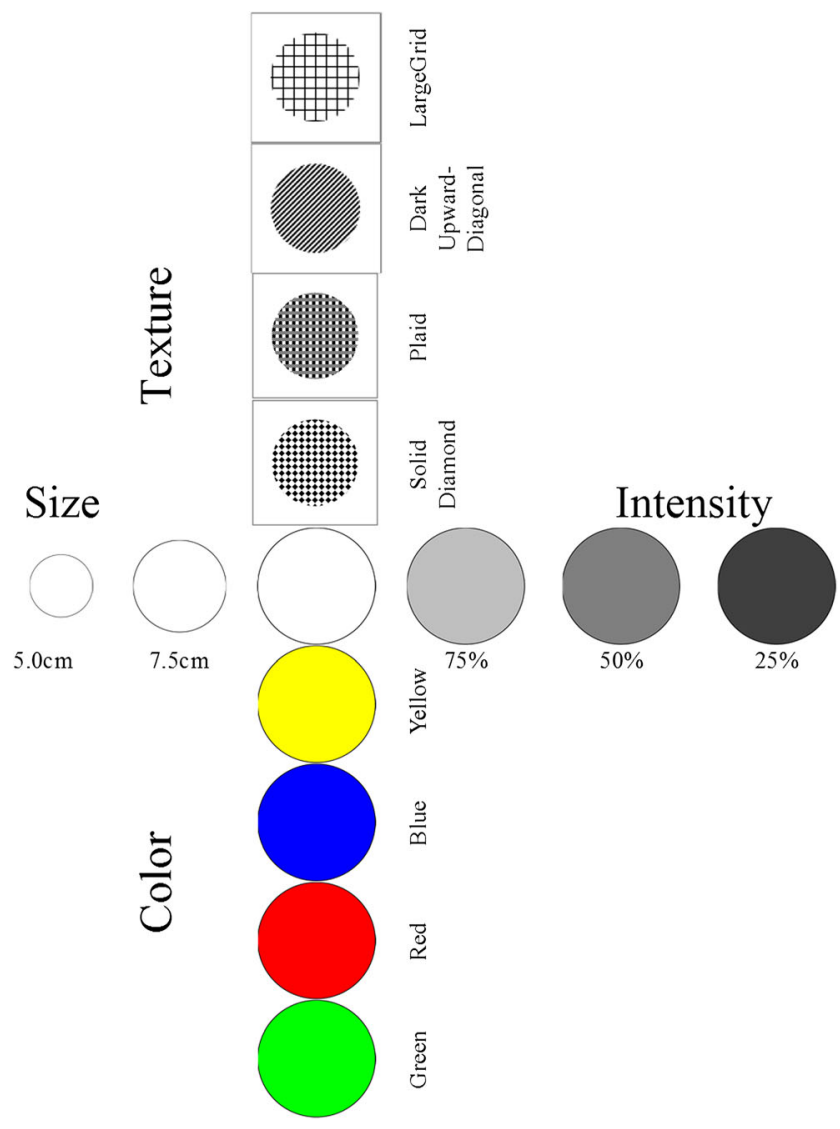

Fig. 4 Stimulus bank. The white center circle was the target during training

$94 \mathrm{~cm}$ from the holding chamber, with the leftmost location $48 \mathrm{~cm}$ from the wall contacting the holding chamber and $10 \mathrm{~cm}$ from the west wall (Fig. 5). Selection of any one of the five presented stimuli resulted in all of the stimuli disappearing, reward delivery, and the presentation of the tone. There were a maximum of 45 trials in each session.

\section{Results and discussion}

Stimulus generalization test A two-way mixed ANOVA conducted on response times with species as a betweensubjects variable and stimulus as a repeated measure revealed a main effect of stimulus, $F(1,14)=2.37$, $p<.001, \eta^{2}=.705$, and a marginal interaction between species and stimulus, $F(1,14)=1.64, p=.077$. For rats, post hoc tests corrected for multiple comparisons using the Bonferroni method comparing novel stimuli to the training stimulus found significantly slower response times for targets of $50 \%$ brightness $[t(6)=2.02$, $p<.05]$ and $25 \%$ size $[t(5)=2.03, p<.05]$ (bottom panel of Fig. 6). For pigeons, post hoc tests corrected for multiple comparisons comparing the novel stimuli to

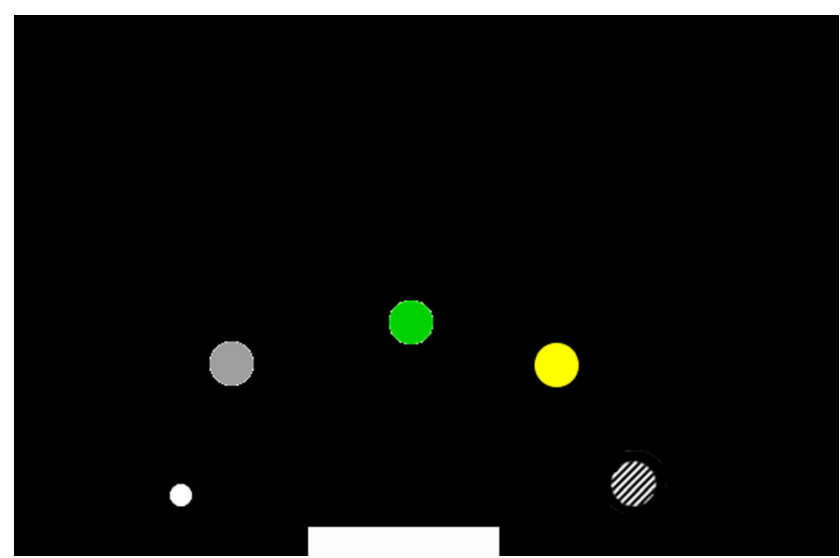

Fig. 5 Experiment 2 preference test diagram. Five stimuli were displayed simultaneously. In this example, the $25 \%$ size, $50 \%$ brightness, green, yellow, and DarkUpwardDiagonal stimuli are used

the training stimulus found significantly slower response times for targets of $25 \%$ brightness $[t(2)=3.83$, $p<.05]$ and $25 \%$ size $[t(2)=3.63, p<.05]$ (top panel of Fig. 6).

Because subjects did not reach the dwell time criterion for reinforcement on every trial during training or testing, we compared the numbers of completed trials for each test stimulus during the individual presentations. A two-way mixed ANOVA conducted on the proportions of trials with each target on which that target was chosen, with species as a between-subjects variable and stimulus as a repeated measure, revealed a main effect of stimulus, $F(1,14)=5.36, p<.001, \eta^{2}$ $=.373$, and a Species $\times$ Stimulus interaction, $F(1,14)=2.36, p$ $<.01, \eta^{2}=.209$. For rats, post hoc tests corrected for multiple comparisons comparing the novel stimuli to the training stimulus found that the following stimuli were chosen significantly less than the training stimulus [25\% brightness, $t(7)=5.22, p$ $<.02$; red, $t(7)=3.84, p<.05$; and $25 \%$ size, $t(7)=3.06, p<$ $0.05]$ (bottom panel of Fig. 7). Pigeons only differed from the training stimuli in the proportion of trials that resulted in a selection for $25 \%$ brightness, $t(3)=3.04, p<.05$ (top panel of Fig. 7).

Feature preference testing To analyze the stimulus preference data, a chi-square test was performed (Fig. 8). When comparing the distribution of target selections to chance, significant differences were found for both rats, $\chi^{2}(1, N=$ $14,671)=798.27, p<.001$, and pigeons, $\chi^{2}(1, N=3,058)=$ $195.59, p<.001$, indicating that choices were not distributed evenly across stimuli. Stimulus-specific comparisons are displayed in Fig. 9.

When comparing the distributions of transfer stimulus target selections to selections of the trained stimulus, significant differences were found in both rats, $\chi^{2}(1, N=14,671)=$ $1,285.54, p<.001$, and pigeons, $\chi^{2}(1, N=3,058)=493.89$, 
Pigeon
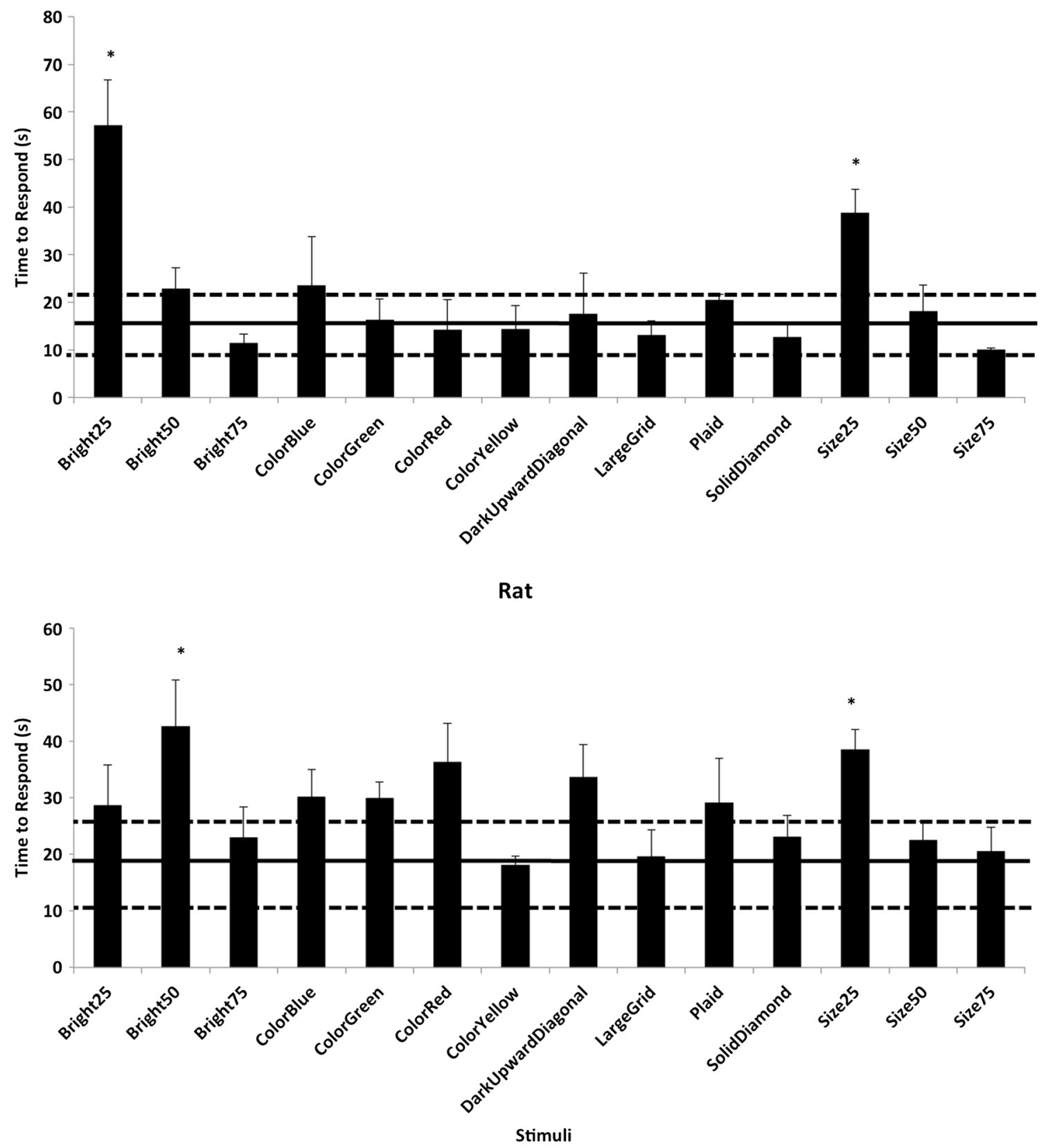

Fig. 6 Mean response times to the different stimuli presented in Experiment 2, stimulus generalization tests. The mean response time to

horizontal dotted lines represent standard errors of the means. Error bars denote $95 \%$ confidence intervals

$p<.001$. Several stimuli were found to differ from the trained stimulus (see Fig. 9).

Both species generalized from the training stimulus to novel stimuli. There were, however, species differences in generalization. Pigeons responded with similar reaction times to the test and novel cues. Nevertheless, pigeon response times did reveal generalization decrements to novel stimuli containing certain feature values. Although the birds were willing to select these stimuli, they were significantly slower for the $25 \%$ size and for $50 \%$ and $75 \%$ brightness, suggesting that these were perhaps less salient when seen from a distance, even though the birds were equally likely to make their selection after the stimuli were located. We cannot explain with certainty why, given the slower response times to $50 \%$ brightness, rats were not slower to respond in the $25 \%$ brightness conditions. This might result from the significantly fewer successful trials involving the stimulus at $25 \%$ brightness. Perhaps subjects failed to detect this faint stimulus on most trials, but on the trials in which they did detect the stimulus, they responded to it with about the same latency as on training trials. Stimuli 
Pigeon

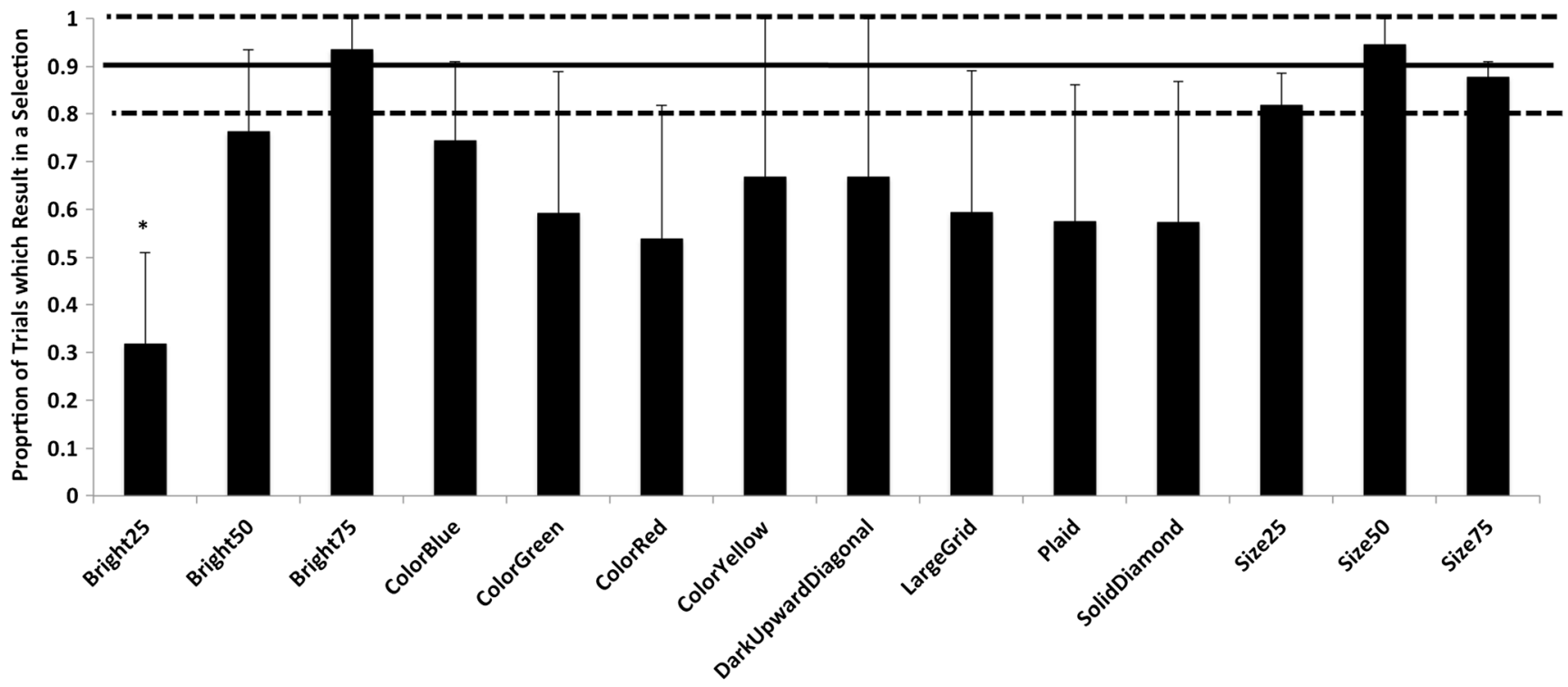

Rat

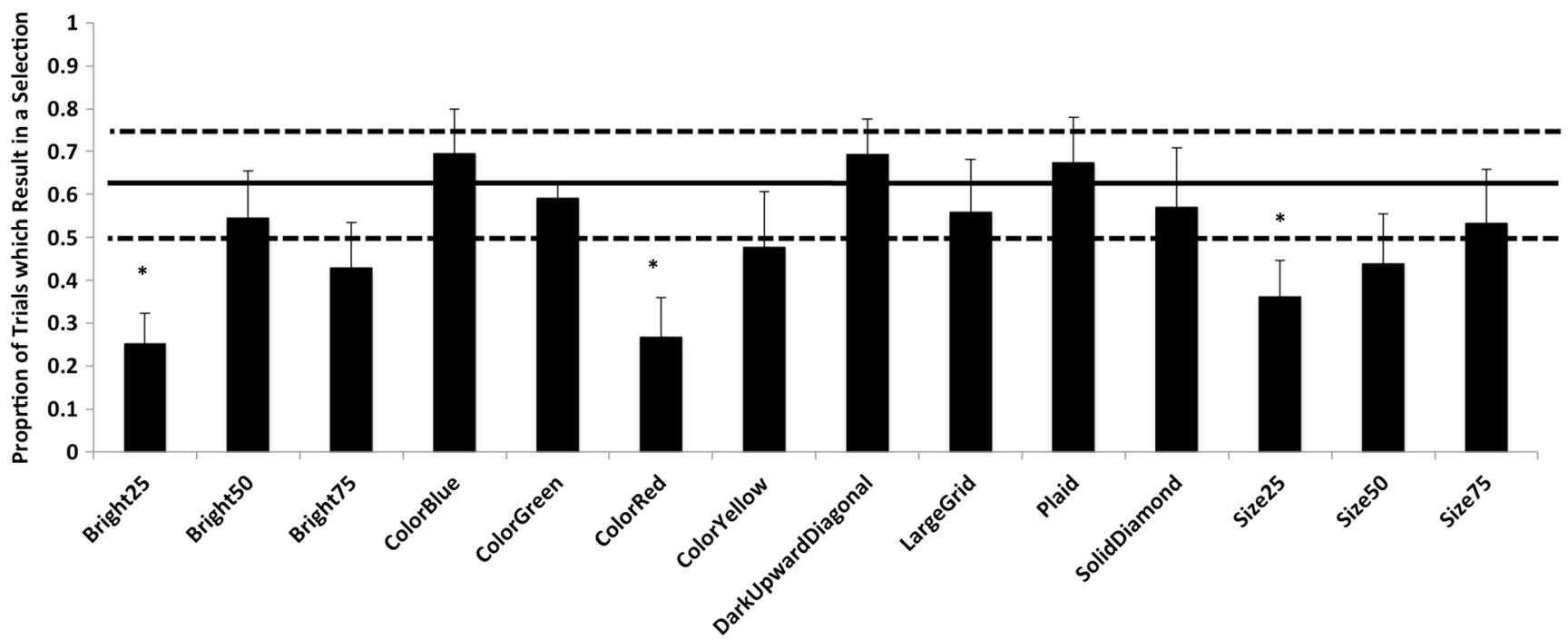

Stimuli

Fig. 7 Mean proportions of trials in the Experiment 2 stimulus generalization test that resulted in a selection being made to each test stimulus. The mean proportions to the training target stimulus are

illustrated by the solid horizontal lines, and the dotted horizontal lines represent standard errors of the means. Error bars denote $95 \%$ confidence intervals

with a brightness of 50\%, however, were responded to much more frequently, providing the opportunity for some longlatency trials to significantly increase the mean response latency to that stimulus.

Rats had poorer generalization, visiting the $25 \%$ and $50 \%$ brightness, red, yellow, dark-upward-diagonal, large-grid, plaid, solid-diamond, and $25 \%$ size stimuli on significantly fewer trials than the trained stimuli. The lower rates of responding to dimmer and smaller stimuli could be partially due to rats' visual system not being as sensitive to dimmer stimuli and longer wavelengths of light (Szél \& Röhlich, 1992), making the color manipulations appear to them similar to the brightness manipulations, and dimmer stimuli in general more difficult to detect. This is supported by the increase in response times to $50 \%$ brightness and $25 \%$ size relative to the trained stimuli.

Because pigeons are more visually guided foragers (Graf, Balsam, \& Silver, 1985) and have heads higher above the ground, it may have been easier for them to see the target locations. The lower number of responses to the pattern stimuli may indicate that this manipulation impaired generalization from previous training in the rats.

The stimulus preference test offered a different view of pigeons' generalization tendencies. Although the pigeons 


\begin{tabular}{|c|c|c|c|c|c|c|c|c|c|c|c|c|c|c|c|c|}
\hline & Species & Bright25 & Bright50 & Bright75 & Blue & Green & Red & Yellow & Train Stim & Diagonal & Grid & Plaid & Diamond & $25 \%$ & $50 \%$ & $75 \%$ \\
\hline \multirow{3}{*}{ Rat } & $x^{2}$ & 124.95 & 73.39 & 6.67 & 23.14 & 4.07 & 119.93 & 12.98 & 57.90 & 60.32 & 27.73 & 42.63 & 105.90 & 112.17 & 25.58 & 0.91 \\
\hline & \begin{tabular}{|l|} 
P-Value \\
\end{tabular} & \begin{tabular}{|l|}
$<0.01$ \\
\end{tabular} & $<0.01$ & 0.01 & $<0.01$ & \begin{tabular}{|l|}
0.04 \\
\end{tabular} & $<0.01$ & $<0.01$ & $<0.01$ & $<0.01$ & $<0.01$ & $<0.01$ & $<0.01$ & $<0.01$ & $<0.01$ & 0.34 \\
\hline & \begin{tabular}{|l|} 
Chance \\
\end{tabular} & Below & \begin{tabular}{|l|} 
Below \\
\end{tabular} & Below & Above & Above & Below & Above & Above & Above & Above & Above & Above & Below & Below & \\
\hline \multirow{3}{*}{ Pigeon } & $x^{2}$ & 38.28 & 1.78 & 53.50 & 0.83 & 5.56 & 5.42 & 4.89 & 33.45 & 1.27 & 3.16 & 0.04 & 0.76 & 18.63 & 7.08 & 20.94 \\
\hline & \begin{tabular}{|l|} 
P-Value \\
\end{tabular} & $<0.01$ & 0.18 & $<0.01$ & 0.36 & $\begin{array}{l}0.02 \\
\end{array}$ & $\begin{array}{l}0.02 \\
\end{array}$ & \begin{tabular}{|l|}
0.03 \\
\end{tabular} & $<0.01$ & 0.26 & \begin{tabular}{|l|}
0.08 \\
\end{tabular} & 0.83 & 0.38 & $<0.01$ & \begin{tabular}{|l|}
0.01 \\
\end{tabular} & $<0.01$ \\
\hline & Chance & Below & & Above & & Below & Below & Below & Above & & Below & & & Below & Above & Above \\
\hline & & & & & & & & & & & & & & & & \\
\hline & & & & & & & & & & & & & & & & \\
\hline & Species & Bright25 & Bright50 & Bright75 & Blue & Green & Red & Yellow & Diagonal & Grid & Plaid & Diamond & $25 \%$ & $50 \%$ & $75 \%$ & \\
\hline \multirow{3}{*}{ Rat } & $x^{2}$ & \begin{tabular}{|l|}
267.87 \\
\end{tabular} & \begin{tabular}{|l}
198.51 \\
\end{tabular} & \begin{tabular}{|l}
78.05 \\
\end{tabular} & 5.90 & \begin{tabular}{|r|}
23.17 \\
\end{tabular} & \begin{tabular}{|r}
262.39 \\
\end{tabular} & \begin{tabular}{|l}
11.76 \\
\end{tabular} & 0.04 & 3.90 & 0.78 & 5.94 & \begin{tabular}{|r|}
250.24 \\
\end{tabular} & 121.41 & \begin{tabular}{|r}
55.59 \\
\end{tabular} & \\
\hline & P-Value & $<0.01$ & $<0.01$ & $<0.01$ & 0.02 & $<0.01$ & $<0.01$ & $<0.01$ & 0.84 & 0.05 & 0.38 & 0.01 & $<0.01$ & $<0.01$ & $<0.01$ & \\
\hline & Trained Stimuli & Less & Less & Less & Less & Less & Less & Less & & Less & & More & Less & Less & Less & \\
\hline \multirow{3}{*}{ Pigeon } & $x^{2}$ & \begin{tabular}{|r|}
98.90 \\
\end{tabular} & 35.33 & 1.31 & 16.72 & 46.01 & 45.52 & 44.53 & 33.17 & 40.30 & 21.83 & 31.19 & 70.98 & 6.94 & 1.18 & \\
\hline & P-Value & $<0.01$ & $<0.01$ & 0.25 & $<0.01$ & $<0.01$ & $<0.01$ & $<0.01$ & $<0.01$ & $<0.01$ & $<0.01$ & $<0.01$ & $<0.01$ & 0.01 & 0.28 & \\
\hline & Trained Stimuli & Below & Below & Above & Below & Below & Below & Below & Below & Below & Below & Below & Below & Below & Below & \\
\hline
\end{tabular}

Fig. 8 Chi-square statistics for rats and pigeons in the Experiment 2 preference test for stimulus characteristics. The top chart compares to chance selections. The bottom chart compares to selection proportions for the trained stimulus

had been willing to respond to a wide variety of stimuli when no alternative was offered, they maintained a strong preference for the trained stimulus when it was present. The similarly high rates of selection to $75 \%$ brightness and $75 \%$ size, with decreased preferences as the brightness or size decreased, indicated that their preference for the trained stimulus emerged through changes along these gradients. The test stimuli most similar to the trained stimulus were selected most often, with increasing discrimination as the test stimulus became more dissimilar. Variations in color and pattern, however, appeared to have been less salient and led to lower proportions of selections.

\section{General discussion}

The experiments reported here demonstrated the utility of the ARENA 2.0 system for comparative open-field experiments. Both avian and mammalian subjects were able to acquire a response in an operant procedure (target training), increase their dwell times until they was easily discriminable from exploratory behavior by the automated tracking system, generalize responding to novel locations (multiple location training), and respond to a variety of visual stimulus transformations (stimulus generalization tests and preference tests). Stimulus presentation and behavioral scoring were automated in a manner similar to other automated operant procedures, such as operant-touchscreen chambers or Skinner boxes, but with the benefits of open-field procedures such as the cheese board or water maze. This system allows for automated openfield operant and Pavlovian testing, greatly increasing the variety of manipulations possible, the number of trials possible per session, and the number of variables recorded, from those of traditional open-field procedures, while reducing the influence of human handling or unintended cues from the experimenter, subject, or test context, such as extramaze cues and order cues.
The ARENA 2.0 system achieved our goals of (a) being able to arbitrarily present stimuli at various physical locations and (b) reliable detection of subject responses to these stimuli among both rats and pigeons. It also has an advantage over the original ARENA system (Badelt \& Blaisdell, 2008) in its ability to present cues at any location on the floor of the test environment instantaneously. Furthermore, the new system eliminates the need for the continual recharging or replacement of batteries, which often failed in the midst of a test session in the original ARENA system. The new system also does not rely on wireless technology or IR beams, reducing interference errors that have often arisen from this technology. By defining a choice response in terms of a minimum criterion dwell time at the target, the system allows for an individual to continue its natural foraging behaviors, such as pecking or sniffing, without requiring different measures to be used for different species. When conducting comparative research, the differences in the forms of experimental methods such as touchscreens and water mazes have led to the development of different measures for different species (Leising \& Blaisdell, 2009). Our system, on the other hand, allows for a single measure, dwell time, that could be influenced by a variety of conditioned responses, such as face washing, orienting to a local cue, or pecking on the ground around the target. Since the target can be located anywhere within the room at any time, preferences for spatial locations can be eliminated easily without the need for extra training. This allows for the dissociation of stimulus attributes such as featural information from location, something very difficult to do with levers or response keys without changing the context. Additionally, because all changes between and within trials, all rewards, and all data recording are handled by computer, many more trials may be recorded per subject in each session. In this study, testing continued for $8 \mathrm{~h}$ a day with minimal involvement and time commitment by the researchers.

As we discussed in the introduction, ARENA 2.0 allows for movement within an open space, and is therefore welladapted to studies of spatial learning. Because ARENA 2.0 

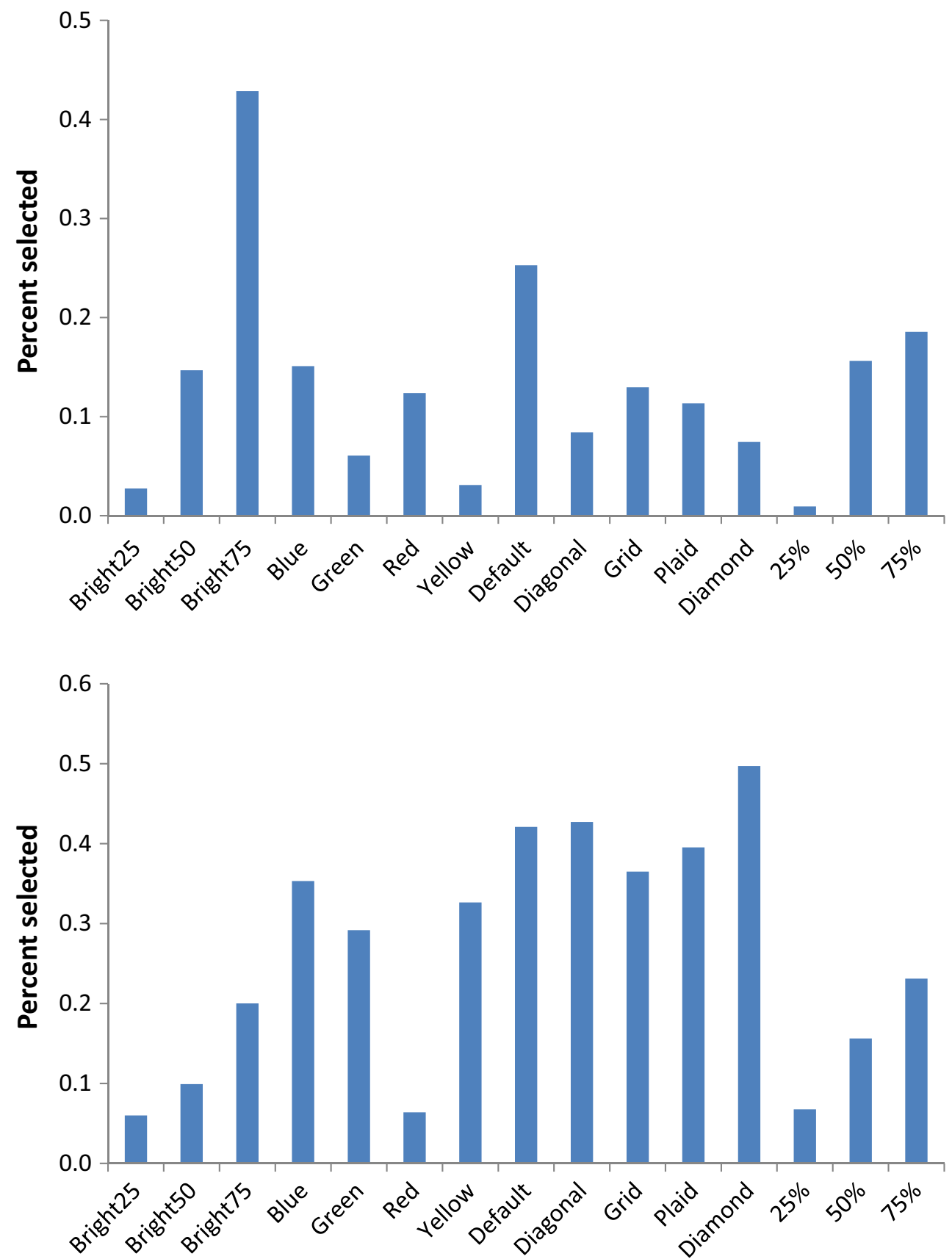

Fig. 9 Mean percentages of stimulus presentations on which that stimulus was selected by pigeons (top panel) and rats (bottom panel) in the preference test of Experiment 2. The dashed lines indicate chance performance, and "Default" indicates the training condition

has the ability to project any desired image, it is a great improvement over the original ARENA system, which was confined to the display of colored lights at discrete locations on the experimental floor (Badelt \& Blaisdell, 2008; Leising et al., 2009). ARENA 2.0 allows for any study that would be conducted on a touchscreen to be performed in a more naturalistic environment. As is illustrated in Experiment 2, both rats and pigeons were able to generalize their responding to a wide array of stimuli, allowing for novel stimuli or stimulus combinations to be introduced, similar to such tests conducted on touchscreens. Our results suggest that the system can be used to assay discrimination training and systematic tests of generalization to novel stimuli, features, or transformations. With these methods, behavioral procedures could be applied for the study of other behavioral and cognitive processes, such as spatial learning and cognition, working and reference memory, perception, and higher-level cognition (e.g., relational discriminations, transitive inferences, numerical cognition and rule learning, etc.). Similar procedures could be adapted to test different species, with modifications to take 
advantage of each species' particular proclivities. For example, we identified (unsurprisingly) that pigeons were better across an array of visual transformations, whereas rats showed poor performance with particular colors.

Author note Support for this research was provided by National Institutes of Health (NIH) Grant NS059076 (A.P.B.) and by National Science Foundation (NSF) Grant BCS-0843027 (A.P.B.). This research was conducted following the relevant ethics guidelines for research with animals and was approved by UCLA's institutional IACUC.

\section{References}

Badelt, S. W., \& Blaisdell, A. P. (2008). Capacitive sensors for detecting proximity and response. Behavior Research Methods, 40, 613-621. doi:10.3758/BRM.40.2.613

Biedermann, T., Garlick, D., \& Blaisdell, A. P. (2012). Food choice in the laboratory pigeon. Behavioural Processes, 91, 129-132.

Blough, D. S. (1986). Odd-item search by pigeons: Method, instrumentation, and uses. Behavior Research Methods, Instruments, \& Computers, 18, 413-419.

Capaldi, E. A., Robinson, G. E., \& Fahrback, S. E. (1999). Neuroethology of spatial learning: The birds and the bees. Annual Review of Psychology, 50, 651-682.

Cook, R. G., Geller, A. I., Zhang, G.-R., \& Gowda, R. (2004). Touchscreen-enhanced visual learning in rats. Behavior Research Methods, Instruments, \& Computers, 36, 101-106.

Cook, R. G., Katz, J. S., \& Blaisdell, A. P. (2012). Temporal properties of visual search in pigeon target localization. Journal of Experimental Psychology: Animal Behavior Processes, 38, 209-216.

Cook, R. G., Shaw, R., \& Blaisdell, A. P. (2001). Dynamic object perception by pigeons: Discrimination of action in video presentations. Animal Cognition, 4, 137-146.

Elsmore, T. F., Parkinson, J. K., \& Mellgren, R. L. (1989). Video touchscreen stimulus-response surface for use with primates. Bulletin of the Psychonomic Society, 27, 60-63.

Graf, J. S., Balsam, P. D., \& Silver, R. (1985). Associative factors and the development of pecking in the ring dove. Developmental Psychobiology, 18, 447-460.

Hauser, M. D., Weiss, D., \& Marcus, G. (2002). RETRACTED: Rule learning by cotton-top tamarins. Cognition, 86, B15-B22.

Leising, K. J., \& Blaisdell, A. P. (2009). Associative basis of landmark learning and integration in vertebrates. Comparative Cognition and Behavior Review, 4, 80-102.
Leising, K. J., Garlick, D., Parenteau, M., \& Blaisdell, A. P. (2009). Behavioral research in pigeons with ARENA: An automated remote environmental navigation apparatus. Behavioural Processes, 81, $105-113$.

Leising, K. J., Garlick, D., \& Blaisdell, A. P. (2011). Overshadowing between landmarks on the touchscreen and in ARENA with pigeons. Journal of Experimental Psychology: Animal Behavior Processes, 37, 488-494.

Leising, K. J., Wong, J., Ruprecht, C. M., \& Blaisdell, A. P. (2014). Blocking between landmarks during 2-D (touchscreen) and 3-D (ARENA) search tasks with pigeons. Learning \& Behavior, 42, 357-364.

Nakagaki, T., Yamada, H., \& Tóth, Á. (2000). Intelligence: Mazesolving by an amoeboid organism. Nature, 407, 470. doi:10. $1038 / 35035159$

Sawa, K., Leising, K. J., \& Blaisdell, A. P. (2005). Sensory preconditioning in spatial learning using a touch screen task in pigeons. Journal of Experimental Psychology: Animal Behavior Processes, 31, 368375.

Scarf, D., Hayne, H., \& Colombo, M. (2011). Pigeons on par with primates in numerical competence. Science, 334, 1664.

Shettleworth, S. (1998). Cognition, behavior, and evolution. Oxford, UK: Oxford University Press.

Skinner, B. F. (1938). The behavior of organisms. New York, NY: Appleton-Century-Crofts.

Skinner, B. F. (1956). A case history in scientific method. American Psychologist, 11, 221-233.

Spetch, M. L., Cheng, K., \& MacDonald, S. E. (1996). Learning the configuration of a landmark array: I. Touch-screen studies with pigeons and humans. Journal of Comparative Psychology, 110, 5568.

Spetch, M. L., Cheng, K., \& Mondloch, M. V. (1992). Landmark use by pigeons in a touch-screen spatial search task. Animal Learning \& Behavior, 20, 281-292.

Stahlman, W. D., Roberts, S., \& Blaisdell, A. P. (2010). Effect of reward probability on spatial and temporal variation. Journal of Experimental Psychology: Animal Behavior Processes, 36, 77-91. doi: $10.1037 / \mathrm{a} 0015971$

Szél, Á., \& Röhlich, P. (1992). Two cone types of rat retina detected by anti-visual pigment antibodies. Experimental Eye Research, 55, 4752.

Young, M. E., Wasserman, E. A., \& Garner, K. L. (1997). Effects of number of items on the pigeon's discrimination of same from different visual displays. Journal of Experimental Psychology: Animal Behavior Processes, 23, 491-501. 